\title{
Metabolomics and metabolic pathway networks from human colorectal cancers, adjacent mucosa, and stool
}

\author{
Dustin G. Brown ${ }^{1}$, Sangeeta Rao ${ }^{2}$, Tiffany L. Weir ${ }^{3}$, Joanne O'Malia ${ }^{4}$, Marlon Bazan", Regina J. Brown ${ }^{5}$
} and Elizabeth P. Ryan ${ }^{1,2^{*}}$

\begin{abstract}
Background: Colorectal cancers (CRC) are associated with perturbations in cellular amino acids, nucleotides, pentose-phosphate pathway carbohydrates, and glycolytic, gluconeogenic, and tricarboxylic acid intermediates. A non-targeted global metabolome approach was utilized for exploring human CRC, adjacent mucosa, and stool. In this pilot study, we identified metabolite profile differences between CRC and adjacent mucosa from patients undergoing colonic resection. Metabolic pathway analyses further revealed relationships between complex networks of metabolites.
\end{abstract}

Methods: Seventeen CRC patients participated in this pilot study and provided CRC, adjacent mucosa $\sim 10 \mathrm{~cm}$ proximal to the tumor, and stool. Metabolomes were analyzed by gas chromatography-mass spectrometry (GC/MS) and ultra-performance liquid chromatography-mass spectrometry (UPLC-MS/MS). All of the library standard identifications were confirmed and further analyzed via MetaboLync ${ }^{T M}$ for metabolic network interactions.

Results: There were a total of 728 distinct metabolites identified from colonic tissue and stool matrices. Nineteen metabolites significantly distinguished CRC from adjacent mucosa in our patient-matched cohort.

Glucose-6-phosphate and fructose-6-phosphate demonstrated 0.64-fold and 0.75-fold lower expression in CRC compared to mucosa, respectively, whereas isobar: betaine aldehyde, $\mathrm{N}$-methyldiethanolamine, and adenylosuccinate had 2.68-fold and 1.88-fold higher relative abundance in CRC. Eleven of the 19 metabolites had not previously been reported for CRC relevance. Metabolic pathway analysis revealed significant perturbations of short-chain fatty acid metabolism, fructose, mannose, and galactose metabolism, and glycolytic, gluconeogenic, and pyruvate metabolism. In comparison to the 500 stool metabolites identified from human CRC patients, only 215 of those stool metabolites were also detected in tissue. This CRC and stool metabolome investigation identified novel metabolites that may serve as key small molecules in CRC pathogenesis, confirmed the results from previously reported CRC metabolome studies, and showed networks for metabolic pathway aberrations. In addition, we found differences between the CRC and stool metabolomes.

Conclusions: Stool metabolite profiles were limited for direct associations with CRC and adjacent mucosa, yet metabolic pathways were conserved across both matrices. Larger patient-matched CRC, adjacent non-cancerous colonic mucosa, and stool cohort studies for metabolite profiling are needed to validate these small molecule differences and metabolic pathway aberrations for clinical application to CRC control, treatment, and prevention.

Keywords: Metabolomics, Colorectal, Cancer, Stool, Metabolites, Tumor, Colon mucosa, Metabolic pathways

\footnotetext{
* Correspondence: E.P.Ryan@colostate.edu

${ }^{1}$ Department of Environmental and Radiological Health Sciences, Colorado

State University, 200 West Lake Street, 1680 Campus Delivery, Fort Collins, CO

80523, USA

${ }^{2}$ Department of Clinical Sciences, Colorado State University, Fort Collins, CO

80523, USA

Full list of author information is available at the end of the article
} 


\section{Background}

Metabolomics has helped cancer research to elucidate specific biomarkers of disease that facilitate personalized therapies and may improve clinical outcomes noninvasively following primary or secondary diagnoses [1]. Colorectal cancer (CRC) is the third leading cause of cancer-related death in the USA [2] and while there are many screening techniques (i.e., colonoscopy, double contrast barium enema, immunochemical-based fecal occult blood test, and serum CEA test [3-5]), high throughput and sensitive molecular tools are needed to support identification of novel biomarkers for metabolic pathway aberrations. Metabolomics is an emerging analytical tool that can be described as the systematic study of the entire profile of small molecules in a clinical sample that are detected using mass spectrometry. Metabolites from a multitude of matrices, such as serum, urine, stool, and tissue, represent the downstream functional products of gene expression and protein synthesis and include environmental exposures and microbial metabolism, all of which influence CRC processes [6]. There remains a gap in knowledge for the spectrum of metabolic pathway alterations that co-exist in the CRC tumor microenvironment which merits a non-targeted, global metabolomics approach [7].

Chan et al. reported 31 metabolites in 2009 that were differentially expressed in paired CRC and adjacent mucosal samples, with glucose levels that were $\sim 67 \%$ higher in the mucosa [8]. In 2014, Qiu et al. identified 15 significantly altered metabolites in matched surgical specimens by comparing CRC and adjacent mucosa from 3 different hospitals in China and 1 in the USA [9]. These metabolite signatures were then used to predict the rate of recurrence and survival in patients after treatment [9]. Most CRCs originate from polyps that exhibit metabolic alterations due to distinct mutations that allow them to replicate uncontrollably, evade host immunity, and become invasive (i.e., Warburg effect, $A P C /$ KRAS/BRAF gene mutations, MSI status) [10-12]. Metabolic fingerprints that could distinguish adjacent mucosa from CRC may reveal stages of pathogenesis, inform the frequency of follow-up screening, and response to preventive measures needed for improved prognosis $[13,14]$.

Paralleled investigations of CRC and adjacent mucosal tissues alongside patient-matched stools that reflect the tumor microenvironment are limited [3]. In the present pilot study, we determined metabolite profile differences between patient-matched CRC and adjacent mucosa and performed metabolic pathway analyses to examine correlations between metabolite expression. We hypothesized that identification of metabolite signatures of colonic tissue that are proximal to and within the CRC represent potential metabolic targets for secondary CRC control, prevention, and treatment. The overlapping metabolites from patient-matched stool and tissue metabolomes as aberrancies in metabolic pathway networks presented herein merit further interrogation for enhanced knowledge of CRC etiology and pathogenesis.

\section{Methods}

\section{Ethics, consent, and permissions}

Seventeen individuals with a scheduled colonic resection after a CRC diagnosis were recruited for this study. All patients provided informed, written consent and knew that data would be used for publication. The patient inclusion criteria for this study included not taking antibiotics prior to surgery (intravenous pre-operative antibiotics were admissible). Tissue and stool samples were collected from Poudre Valley Hospital (PVH; Fort Collins, CO, USA) and de-identified for personal information before processing at Colorado State University (protocol nos. 10-1006 and 10-1670H).

\section{Sample collection}

Within 30 min of surgery, a 5-mm section of CRC $(n=16)$ and adjacent mucosal tissue samples that were $\sim 10 \mathrm{~cm}$ proximal to the CRC $(n=17)$ were collected from resected colons in the PVH Pathology Lab. Human tissue samples were stored immediately at $-80{ }^{\circ} \mathrm{C}$ following collection until processed for metabolomics. Stool samples were selfcollected by patients just prior to surgery prep in pre-labeled, coded containers by the patients and stored at $-80{ }^{\circ} \mathrm{C}$ immediately $(n=13)$. Collection was verified by the study technician who was present in the pathology lab and pre-op room for each resection. Patientmatched CRC, adjacent mucosa, and stool were collected for all individuals with the following exceptions: one patient was missing a CRC sample but had an adjacent mucosa and stool sample, and four patients provided mucosa and CRC but did not provide stool. All samples were shipped to Metabolon, Inc. (Durham, NC, USA) for metabolomics and multivariate statistical analyses. Table 1 shows human patient and tumor characteristics associated with tissue samples analyzed herein [15].

\section{Sample accessioning and preparation}

Tissue and stool metabolite extractions for GC-MS and UPLC-MS/MS were completed by Metabolon Inc. Both platforms were chosen to provide broad, non-targeted detection of metabolites. Each sample was accessioned into the Metabolon Laboratory Information Management System (LIMS) and assigned a unique identifier associated with the original de-identified study code number. This identifier was used to track all sample handling, tasks, and results. All samples were maintained at $-80{ }^{\circ} \mathrm{C}$ until analysis [16]. 
Table 1 Colorectal cancer patient and tumor characteristics

\begin{tabular}{|c|c|}
\hline Age (years) [mean \pm SD] & $58.8 \pm 13.8$ \\
\hline \multicolumn{2}{|l|}{ Sex } \\
\hline Males & $13(76 \%)$ \\
\hline Females & $4(24 \%)$ \\
\hline BMI $\left(\mathrm{kg} / \mathrm{m}^{2}\right)[$ mean $\pm \mathrm{SD}]$ & $29.3 \pm 4.7$ \\
\hline \multicolumn{2}{|l|}{ Tumor stage } \\
\hline Тх/T0 & $1(6 \%)$ \\
\hline Tis & $1(6 \%)$ \\
\hline $\mathrm{T} 1$ & $3(18 \%)$ \\
\hline $\mathrm{T} 2$ & $3(18 \%)$ \\
\hline T3 & $8(46 \%)$ \\
\hline $\mathrm{T} 4$ & $1(6 \%)$ \\
\hline \multicolumn{2}{|l|}{ Tumor location } \\
\hline Cecum & $1(6 \%)$ \\
\hline Ascending & $5(31 \%)$ \\
\hline Descending & $1(6 \%)$ \\
\hline Sigmoid & $6(38 \%)$ \\
\hline Rectum & $3(19 \%)$ \\
\hline \multicolumn{2}{|l|}{ Tumor size $(\mathrm{cm})$} \\
\hline $0<2$ & $6(35 \%)$ \\
\hline $2<4$ & 5 (29 \%) \\
\hline $4<6$ & $3(18 \%)$ \\
\hline $6<8$ & $3(18 \%)$ \\
\hline \multicolumn{2}{|l|}{ Tumor grade } \\
\hline Low & $15(94 \%)$ \\
\hline High & $1(6 \%)$ \\
\hline
\end{tabular}

Samples were extracted using the automated MicroLab STAR $^{\bullet}$ robotics system from Hamilton Company (Reno, NV, USA). A set of recovery standards, tridecanoic acid, chloro-phenylalanine, D6-cholesterol, and fluorophenylglycine was added prior to the first step in the extraction process for quality control purposes, with final extraction standard concentrations ranging from 2.5 to $25 \mu \mathrm{g} / \mathrm{mL}$. Sample preparation was conducted using a methanol extraction to remove the protein fraction while allowing the maximum recovery of small molecules. Tissue samples were homogenized in water $(5 \mu \mathrm{L} / \mathrm{mg}$ of sample) using a GenoGrinder 2000 bead grinder (Glen Mills, Clifton, NJ, USA), shaking for $5 \mathrm{~min}$ at 1000 strokes per minute at room temperature. Methanol extraction utilized five volumes of methanol (5:1 methanol/water) with vigorous shaking at room temperature for $2 \mathrm{~min}$ followed by centrifugation at $680 \times g$ for $3 \mathrm{~min}$. The resulting methanol extract was divided into four fractions: one for analysis by UPLC-MS/ MS with positive ion mode electrospray ionization and one for negative ion mode electrospray ionization, one for analysis by GC-MS, and one sample was reserved for backup. Samples were placed briefly on a TurboVap ${ }^{\oplus}$ from Zymark Corporation (Hopkinton, MA, USA) to remove the organic solvent. The samples were stored overnight under nitrogen before preparation for UPLC-MS/MS and each sample was dried under vacuum for a minimum of $18 \mathrm{~h}$ before derivatization for GC-MS analysis. Samples were analyzed in concert with several types of controls to allow instrument performance monitoring and aid chromatographic alignment.

\section{GC-MS analysis}

Samples were derivatized under nitrogen using bistrimethyl-silyltrifluoroacetamide and separated on a $5 \%$ diphenyl/95 \% dimethyl polysiloxane-fused silica column $(20 \mathrm{~m} \times 0.18 \mathrm{~mm}$ ID; $0.18-\mu \mathrm{m}$ film thickness) with helium as carrier gas and a temperature ramp from 60 to $340{ }^{\circ} \mathrm{C}$ in a 17.5 -min period. Internal standards amylbenzene, 1-phenylhexane, 1-phenyloctane, 1phenyldecane, 1-phenyldodecane, hexadecylbenzene, octadecylbenzene, tetradecylbenzene, and 2,6-di-tert-butyl-4methylphenol were added to each sample (250 ng of each standard per sample). Samples were analyzed on a Thermo-Finnigan Trace DSQ fast-scanning singlequadrupole mass spectrometer using electro-impact ionization (EI) and operated at unit mass resolving power. The scan range was from $50-750 \mathrm{~m} / \mathrm{z}$.

\section{UPLC-MS/MS analysis}

The UPLC-MS/MS portion of the platform was based on a Waters ACQUITY UPLC and a Thermo-Finnigan LTQ MS operated at nominal mass resolution, which consisted of an electrospray ionization (ESI) source and linear ion-trap (LIT) mass analyzer. The dried sample extract was reconstituted in acidic or basic UPLCcompatible solvents, each of which contained 11 to 13 injection standards at fixed concentrations [17]. One aliquot was analyzed using acidic positive ion-optimized conditions and the other using basic, negative ionoptimized conditions in two independent injections using separate dedicated columns (Waters UPLC BEH C18-2.1 $\times 100 \mathrm{~mm}, 1.7 \mu \mathrm{m})$. Extracts reconstituted in acidic conditions were gradient eluted using water and methanol containing $0.1 \%$ formic acid, while the basic extracts, which also used water/methanol, contained $6.5 \mathrm{mM}$ ammonium bicarbonate. The MS analysis alternated between MS and data-dependent MS/MS scans using dynamic exclusion and the scan range was from $80-1000 \mathrm{~m} / \mathrm{z}$.

\section{Data extraction and compound identification}

Raw data was extracted and peak-identified as previously described [14]. Biochemical identifications were based on (1) retention index within a narrow RI window of the 
proposed identification, (2) accurate mass match to the library +/- $0.005 \mathrm{amu}$, and (3) the MS/MS forward and reverse scores between the experimental data and authentic standards. The MS/MS scores were based on a comparison of the ions present in the experimental spectrum to the ions present in the library spectrum. More than 3300 commercially available purified standard compounds have been acquired and registered into LIMS for distribution to both the UPLC-MS/MS and GC-MS platforms for determination of their analytical characteristics [16]. All detected metabolites were identified with level 1 or 2 (noted by an asterisk symbol) confidence of identification [18].

\section{Metabolomics statistical analysis}

Metabolite profiles in CRC patients were quantified in terms of relative abundance and median scaled to 1 . Following $\log$ transformation and imputation of missing values, if any, with the minimum observed value for each compound imputed, statistical analyses were performed to identify significant differences between experimental groups. Statistical analyses were performed in ArrayStudio (Omicsoft, Cary, NC, USA), R version 2.14.2, and/or SAS v9.4 [19]. Metabolite profile distinctions between CRC and adjacent mucosa were evaluated by matched pair $t$ tests. An estimate of the false discovery rate ( $q$ value) was calculated to take into account the multiple comparisons that normally occur in metabolomic-based studies. A $q$ value threshold of $\leq 0.10$ was used to correct for false discovery of statistically significant compounds due to multiple hypothesis testing. Metabolites with $q$ values that exceeded this threshold were discarded from further analysis. Fold difference (FD) was determined by dividing the relative abundance of the metabolite in the CRC by the relative abundance of the metabolite in the adjacent mucosa. Metabolites with $p$ values of $\leq 0.05$ with $q$ values below the threshold of $\leq 0.10$ were considered statistically significant in this study.

\section{Metabolic pathway networks and analysis}

To visualize and analyze small molecules within relevant networks of metabolic pathways, the detected metabolites in CRC and adjacent mucosa were subjected to MetaboLync pathway analysis (MPA) software (portal.metabolon.com). Significantly altered pathways were determined by completing pathway set enrichment analysis within MPA software which was determined by the following equation:

\# of significant metabolites in pathway $(k) /$ total \# of detected metabolites in pathway $(m) /$ total \# of significant metabolites $(n) /$ total \# of detected metabolites $(N)$ or $(k / m) /(n / N)$.

A pathway impact score greater than one indicates that the pathway contains a higher number of experimentally regulated compounds relative to the overall study in CRC than adjacent mucosa, suggesting that the pathway may be of interest to the metabolite perturbations observed. Finally, the significantly altered pathways and metabolites were visualized via a Pathway Visualizations tool using Cytoscape v 2.8.3 software [20].

\section{Results \\ Metabolite differences between CRC and adjacent mucosa}

Principal component analysis (PCA) of CRC and adjacent mucosa samples revealed no distinct clustering by tissue type (Fig. 1a). $z$-scores for 19 metabolites significantly different between CRC and adjacent mucosa are illustrated in Fig. 1b. Table 2 lists the metabolites and metabolic pathways with significant differences between $\mathrm{CRC}$ and adjacent mucosa in 17 patients $(p \leq 0.05)$. Data are presented as the mean fold difference in CRC metabolite abundance compared to adjacent mucosa for all patients. The 5 metabolites that showed higher abundance in CRC includes isobar: betaine aldehyde, $\mathrm{N}$-methyldiethanolamine (2.68-fold) representing glycine, serine, and threonine metabolic pathways, adenylosuccinate (1.88-fold) representing the purine (adenine containing) metabolic pathway, isovalerate (1.45-fold) representing leucine, isoleucine, and valine metabolic pathways, valerate (1.37-fold) representing the short-chain fatty acid metabolic pathway, and N1-methyl-2-pyridonecarboxamide (1.29-fold) representing nicotinate and nicotinamide metabolic pathways. Table 2 further shows the 14 metabolites with lower abundance in CRC compared to mucosa. These include 2 -aminoadipate $(0.93$-fold) representing the lysine metabolic pathway, stearoyl sphingomyelin (0.9-fold) representing the sphingolipid metabolic pathway, 4-hydroxyphenylpyruvate (0.88-fold) representing phenylalanine and tyrosine metabolic pathways, sorbitol (0.87-fold) representing fructose, mannose, and galactose metabolic pathways, alpha-hydroxyisovalerate (0.86-fold) representing leucine, isoleucine, and valine metabolic pathways, cys-gly, oxidized (0.84-fold) representing the glutathione metabolic pathway, trytophylglycine (0.84-fold), aspartylvaline (0.81-fold), and aspartyltryptophan (0.76-fold) representing the dipeptide metabolic pathway, deoxycholate (0.84-fold) and 7-ketodeoxycholate (0.81-fold) representing secondary bile acid metabolism, asparagine $(0.81$-fold $)$ representing alanine and aspartate metabolic pathways, and glucose-6-phosphate (0.64-fold) and fructose-6-phosphate $(0.75$-fold) representing glycolytic, gluconeogenic, and pyruvate metabolic pathways. The information on the metabolic pathway, metabolite name, platform of detection, Kyoto Encyclopedia of Genes and Genomes (KEGG), Human Metabolome DataBase (HMDB), and PubChem identifiers for all 19 metabolites is listed in Additional file 1: Table S1. 
a

b

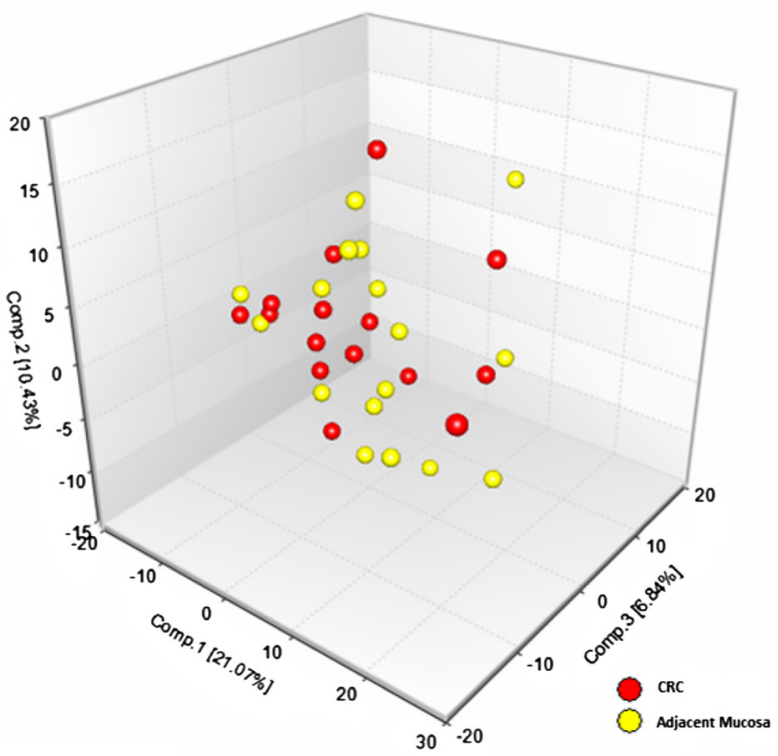

Isobar: betaine aldehyde, $\mathrm{N}$-methyldiethanolamine Asparagine 2-aminoadipate 4-hydroxyphenlpyruvate Isovalerate Alpha-hydroxyisovalerate

Cys-gly, oxidized Aspartyltryptophan Aspartylvaline Tryptophylglycine Glucose-6-phosphate Fructose-6-phosphate Sorbito Valerate Stearoyl Sphingomyelin Deoxycholate 7-ketodeoxycholate

Adenylosuccinate N1-methyl-2-pyridone5-carboxamide

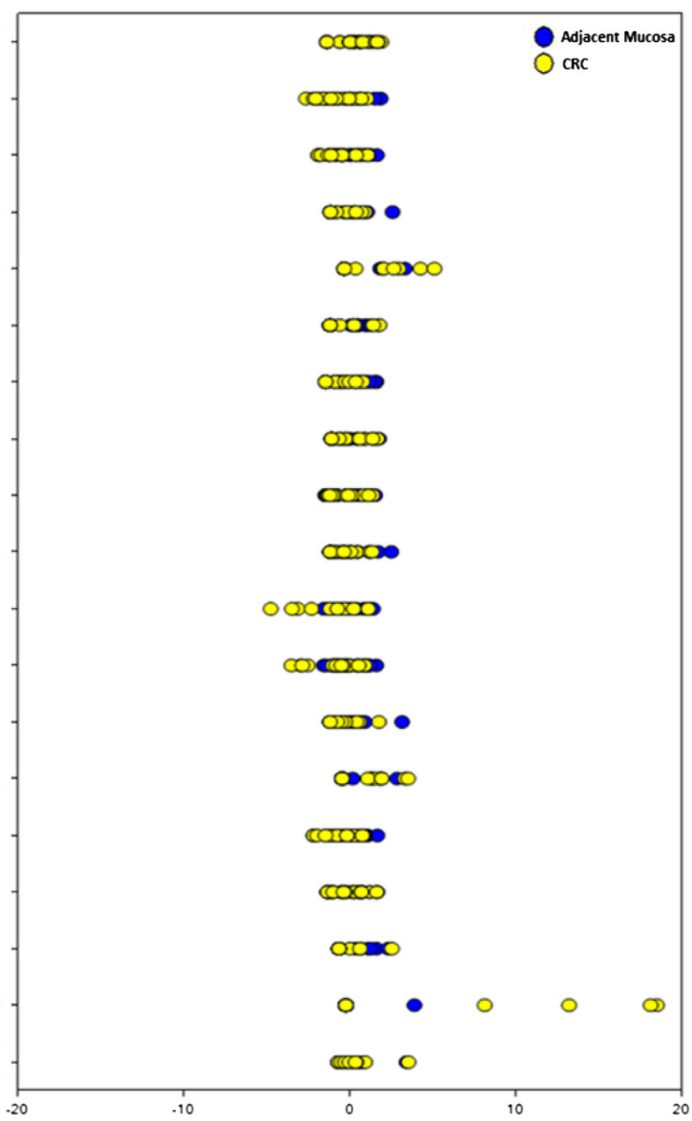

Fig. 1 Metabolomics analysis of CRC and adjacent mucosa. a PCA CRC and adjacent mucosa and $\mathbf{b} z$-scores for the 19 significantly different metabolites and varied expression between CRC and adjacent mucosa

Metabolic network differences between CRC and adjacent mucosa

The pathway set enrichment analysis was performed to elucidate the metabolic pathways affected by metabolite distinctions between CRC and adjacent mucosa. This analysis revealed significant perturbation of 14 metabolic networks, including but not limited to short-chain fatty acid (23.32), fructose, mannose, and galactose (5.83), and glycolytic, gluconeogenic, and pyruvate (5.18) metabolic pathways (Fig. 2). The Cytoscape Pathway Classification 
Table 2 Metabolites with statistically significant differences between colorectal cancer tissue and adjacent mucosa

\begin{tabular}{llll}
\hline Metabolic pathway & Metabolite & $p$ value & Fold difference (CRC/mucosa) \\
\hline Glycine, serine, and threonine metabolism & Isobar: betaine aldehyde, N-methyldiethanolamine & 0.015 & $2.68 \uparrow$ \\
Purine metabolism, adenine containing & Adenylosuccinate & 0.03 & $1.88 \uparrow$ \\
Leucine, isoleucine, and valine metabolism & Isovalerate & 0.0073 & $1.45 \uparrow$ \\
Short-chain fatty acid & Valerate & 0.013 & $1.37 \uparrow$ \\
Nicotinate and nicotinamide metabolism & N1-methyl 2-pyridone-5-carboxamide & 0.018 & $1.29 \uparrow$ \\
Lysine metabolism & 2-aminoadipate & 0.043 & $0.93 \downarrow$ \\
Sphingolipid metabolism & Stearoyl sphingomyelin & 0.049 & $0.9 \downarrow$ \\
Phenylalanine and tyrosine metabolism & 4-hydroxyphenylpyruvate & 0.05 & $0.88 \downarrow$ \\
Fructose, mannose, and galactose metabolism & Sorbitol & 0.029 & $0.87 \downarrow$ \\
Leucine, isoleucine, and valine metabolism & Alpha-hydroxyisovalerate & 0.037 & $0.86 \downarrow$ \\
Glutathione metabolism & Cys-gly, oxidized & 0.047 & $0.84 \downarrow$ \\
Dipeptide & Tryptophylglycine & 0.041 & $0.84 \downarrow$ \\
Secondary bile acid metabolism & Deoxycholate & 0.045 & $0.84 \downarrow$ \\
& 7-ketodeoxycholate & 0.04 & $0.81 \downarrow$ \\
Alanine and aspartate metabolism & Asparagine & 0.025 & $0.81 \downarrow$ \\
Dipeptide & Aspartylvaline & 0.035 & $0.81 \downarrow$ \\
Glycolysis, gluconeogenesis, and pyruvate metabolism & Fructose-6-phosphate & 0.019 & $0.76 \downarrow$
\end{tabular}

$\uparrow$ designates metabolites with significantly $(p \leq 0.05)$ higher expression in CRC when compared with adjacent mucosa (metabolite ratio of $\geq 1.00)$ and $\downarrow$ designates metabolites with significantly $(p \leq 0.05)$ higher expression in adjacent mucosa when compared with CRC (metabolite ratio of $<1.00)$

Network view of impacted metabolic networks for lipid, carbohydrate, amino acid, and cofactors and vitamin pathway metabolites are shown in Fig. 3. Figure 3 shows the following CRC discriminatory metabolites in their respective metabolic network. These are stearoyl sphingomyelin (sphingolipid metabolism), valerate (short-chain fatty acid metabolism), 7-ketodeoxycholate and deoxycholate (secondary bile acid metabolism), fructose-6-phosphate and glucose-6-phosphate (glycolysis, gluconeogenesis, and pyruvate metabolism), sorbitol (fructose, mannose, and galactose metabolism), 4-hydroxyphenylpruvate (phenylalanine and tyrosine metabolism), isobar: betaine

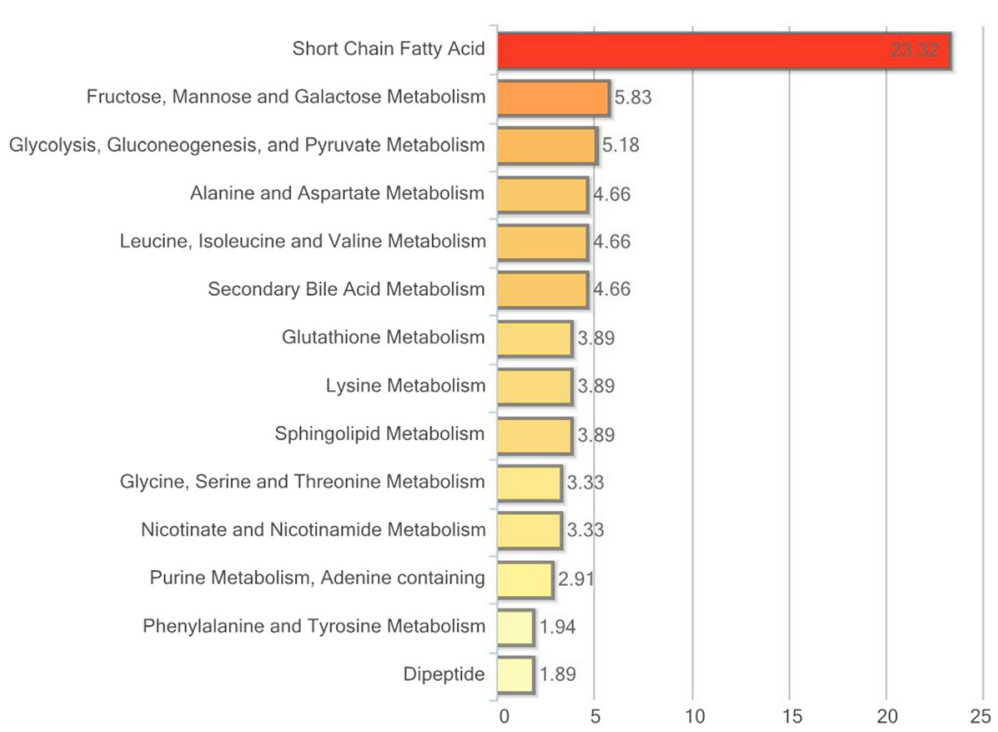

Fig. 2 Pathway set enrichment of metabolic networks distinguished between CRC and adjacent mucosa. There were 14 pathway distinctions determined by a pathway impact score greater than 1. The pathway impact score was determined as defined in the "Methods" section 


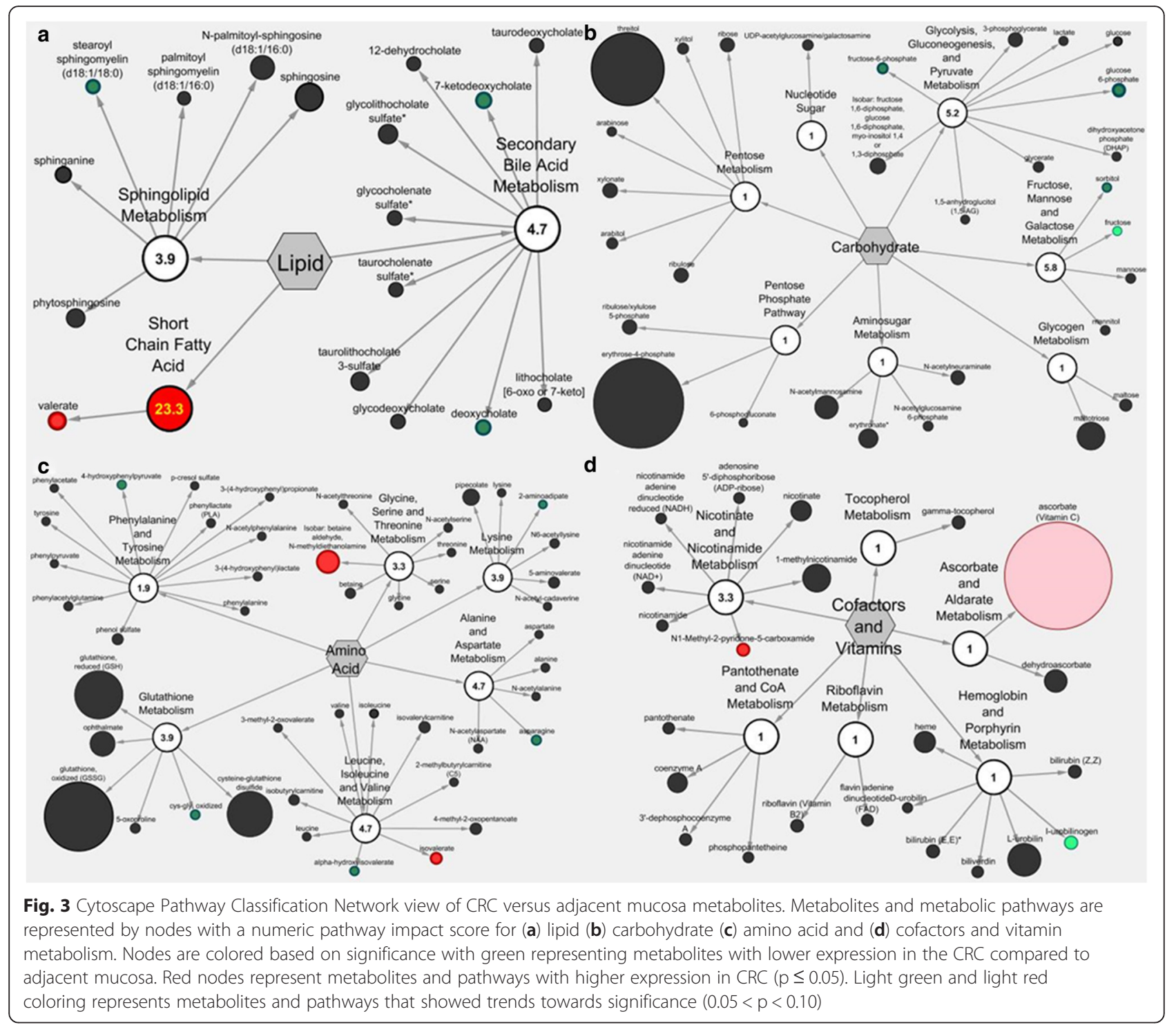

aldehyde, N-methyldiethanolamine (glycine, serine, and threonine metabolism), 2-aminoadipate (lysine metabolism), asparagine (alanine and aspartate metabolism), cys-gly, oxidized (glutathione metabolism), alphahydroxyisovalerate and valerate (leucine, isoleucine, and valine metabolism), and N1-methyl-2-pyridone-5-carboxamide (nicotinate and nicotinamide metabolism).

\section{Stool metabolome of CRC patients}

In this study, we detected 500 metabolites in the stool matrix of $13 \mathrm{CRC}$ patients. The metabolic pathway, metabolite name, platform of detection, KEGG, HMDB, and PubChem identifiers of all detected stool metabolites can be found in Additional file 1: Table S2. This includes 102 amino acid metabolites representing alanine and aspartate, glutamate, glutathione, glycine, serine, and threonine, histidine, leucine, isoleucine, and valine, lysine, methionine, cysteine, SAM, and taurine, phenylalanine and tyrosine, polyamine, tryptophan, and urea cycle; arginine and proline metabolic pathways. It also included 27 carbohydrate metabolites representing aminosugar, disaccharides and oligosaccharides, fructose, mannose, and galactose, glycogen, glycolysis, gluconeogenesis, and pyruvate, pentose, and metabolic pathways. Twenty cofactors and vitamin metabolites representing ascorbate and aldarate, hemoglobin and porphyrin, nicotinate and nicotinamide, pantothenate and CoA, riboflavin, tetrahydrobiopterin, thiamine, tocopherol, and vitamin B6 metabolic pathways were identified. Additionally, we observed 7 energy metabolites in the stool metabolome including oxidative phosphorylation and TCA cycle intermediates. The largest portion of the CRC stool metabolome were metabolites involved in lipid metabolism representing carnitine, endocannabinoid, 
fatty acid, fatty alcohol, long chain, glycerolipid, inositol, ketone bodies, lysolipid, monoacylglycerol, phospholipid, primary and secondary bile acid, sphingolipid, steroid, and sterol metabolic pathways. There were 25 nucleotide metabolites representing purine and pyrimidine metabolism, 106 peptide metabolites representing dipeptide, gamma-glutamyl amino acid, and polypeptide metabolism, and 71 xenobiotics representing chemical, drug, food component/plant and xanthine metabolic pathways (Table 3).

\section{Metabolome overlap and comparison between CRC and stool}

We next identified the metabolome overlap and differences across CRC, adjacent mucosa, and stool. A total of 728 small molecules were detected in CRC, adjacent mucosa, and stool collected from recently diagnosed CRC patients undergoing colonic resection. Compared to the 19 discriminatory metabolites between CRC and adjacent mucosa, only 7 of these discriminatory metabolites were also detected in the stool metabolome. These metabolites were alphahydroxyisovalerate, isovalerate, N1-methyl-2-pyridine5-carboxamide, 7-ketodeoxycholate, deoxycholate, valerate, and tryptophylglycine. The Venn diagram in Fig. 4 shows the 213 metabolites that were common to all 3 sample matrices when compared to the 728 total metabolites detected. Hydrochlorothiazide and pacetamidophenylglucuronide were identified only in CRC whereas mannitol was specific to adjacent mucosa. There were 285 unique stool metabolites, of which 214 stool metabolites overlapped in mucosa, and 214 metabolites shared between stool and CRC. 7-beta-hydroxycholesterol and 2-oxindole-3-acetate were uniquely shared between stool and CRC and stool and mucosa, respectively. Additional file 1: Figure S1 further illustrates the distinct and overlapping tissue and stool metabolites of gut microbial and host origins [21].

\section{Discussion}

This multi-platform CRC and adjacent mucosa metabolome analysis led to the identification of 11 novel metabolites that have not previously been detected for CRC relevance in animals or humans. Adenylosuccinate, isovalerate, valerate, N1-methyl-2-pyridone-5-carboxamide, stearoyl sphingomyelin, 4-hydroxyphenylpyruvate, aspartylvaline, aspartyltryptophan, tryptophyglycine, glucose-6-phosphate, and fructose-6-phosphate merit further investigation as key small molecules in CRC pathogenesis. It is noteworthy that tryptophylglycine was detected in a fecal metabolomics study comparing CRC patients and matched healthy controls [22], whereas our report is the first to our knowledge to show tryptophylglycine metabolite levels in human tissue. One limitation of this study is the small sample size and subsequent low representation of each tumor stage and location. A strength of this pilot investigation was the capacity to obtain CRC and adjacent mucosa with stool samples from the same patient and utilize a multi-platform analytical approach that is comparable to other human datasets (GC/LC-MS) $[8,9,23]$.

Our findings supported the identification of 8 metabolites that were discriminatory between CRC and adjacent mucosa in previous reports and included 2-aminoadipate, isobar: betaine aldehyde, N-methyldiethanolamine, alpha-hydroxyisovalerate, cys-gly, oxidized, deoxycholate, 7-ketodeoxycholate, sorbitol, and asparagine [9, 14, 23]. However, these results were not consistent by the relative abundance, such that the asparagine, 2aminoadipate, alpha-hydroxyisovalerate, deoxycholate, 7-ketodeoxycholate, sorbitol and cys-gly, oxidized had higher expression in adjacent mucosa compared to CRC, whereas isobar: betaine aldehyde, Nmethyldiethanolamine had higher expression in CRC than in adjacent mucosa in this study [14]. Intraindividual differences in tumor heterogeneity and analytical platforms may, in part, explain these discrepancies [11].

Two secondary bile acid metabolites (7-ketodeoxycholate and deoxycholate) showed higher expression in adjacent mucosa compared with $\mathrm{CRC}$ and were detectable across sample matrices (Additional file 1: Table S1). These metabolites are associated with generation of reactive oxygen and nitrogen species [24-27], altering the stability of the cell and mitochondrial membranes $[25,26]$, and inducing oxidative DNA damage $[25,26]$, mutation $[25,26]$, and apoptosis in CRC $[25,26]$. Deoxycholic acid was shown to be diet-modifiable in the plasma metabolome [28], and had higher expression in adjacent mucosa in this study. Alternatively, the variable levels we observed between tissue matrices could be due to gut microbial metabolism. The gut mircobiota are known to convert primary bile acids to secondary bile acids, a process that may be $\mathrm{pH}$-dependent [29]. Variable gut microbial populations throughout the colon and from person to person may also contribute to the differential expression of secondary bile acids observed in this study (Fig. 3a).

Two additional key small molecules of CRC pathogenesis were elucidated in this global, metabolite profiling: N1-methyl-2-pyridone-5-carboxamide and sorbitol. These small molecules are important because they were discriminatory between CRC and adjacent mucosa in this study and they have been reported in disease contexts including chronic renal failure and diabetes [30, 31]. N1-methyl 2-pyridone-5-carboxamide (N125) 
Table 3 CRC stool metabolite numbers within each of the assigned metabolic pathways

\begin{tabular}{|c|c|}
\hline Metabolic pathway & Number of metabolites \\
\hline Amino acid & 102 \\
\hline Alanine and aspartate & 5 \\
\hline Glutamate & 4 \\
\hline Glutathione & 1 \\
\hline Glycine, serine, and threonine & 6 \\
\hline Histidine & 5 \\
\hline Leucine, isoleucine, and valine & 17 \\
\hline Lysine & 8 \\
\hline Methionine, cysteine, SAM, and taurine & 9 \\
\hline Phenylalanine and tyrosine & 23 \\
\hline Polyamine & 6 \\
\hline Tryptophan & 10 \\
\hline Urea cycle; arginine and proline & 8 \\
\hline Carbohydrate & 27 \\
\hline Aminosugar & 6 \\
\hline Disaccharides and oligosaccharides & 2 \\
\hline Fructose, mannose, and galactose & 4 \\
\hline Glycogen & 1 \\
\hline Glycolysis, gluconeogenesis, and pyruvate & 3 \\
\hline Pentose & 11 \\
\hline Cofactors and vitamins & 20 \\
\hline Ascorbate and aldarate & 1 \\
\hline Hemoglobin and porphyrin & 5 \\
\hline Nicotinate and nicotinamide & 4 \\
\hline Pantothenate and CoA & 1 \\
\hline Riboflavin & 2 \\
\hline Tetrahydrobiopterin & 1 \\
\hline Thiamine & 2 \\
\hline Tocopherol & 3 \\
\hline Vitamin B6 & 1 \\
\hline Energy & 7 \\
\hline Oxidative phosphorylation & 1 \\
\hline TCA cycle & 6 \\
\hline Lipid & 142 \\
\hline Carnitine metabolism & 3 \\
\hline Endocannabinoid & 2 \\
\hline Fatty acid & 56 \\
\hline Fatty alcohol, long chain & 2 \\
\hline Glycerolipid & 2 \\
\hline Inositol & 2 \\
\hline Ketone bodies & 1 \\
\hline Lysolipid & 16 \\
\hline Monoacylglycerol & 8 \\
\hline
\end{tabular}

Table 3 CRC stool metabolite numbers within each of the assigned metabolic pathways (Continued)

\begin{tabular}{|c|c|}
\hline Phospholipid & 1 \\
\hline Primary bile acid & 6 \\
\hline Secondary bile acid & 12 \\
\hline Sphingolipid & 4 \\
\hline Steroid & 9 \\
\hline Sterol & 7 \\
\hline Nucleotide & 25 \\
\hline Purine and pyrimidine & 1 \\
\hline Purine metabolism & 5 \\
\hline (Hypo) xanthine/inosine containing & 2 \\
\hline Purine metabolism, adenine containing & 4 \\
\hline Purine metabolism, guanine containing & 1 \\
\hline Pyrimidine metabolism, cytidine containing & 1 \\
\hline Pyrimidine metabolism, orotate containing & 4 \\
\hline Pyrimidine metabolism, thymine containing & 7 \\
\hline \multicolumn{2}{|l|}{ Pyrimidine metabolism, uracil containing } \\
\hline Peptide & 106 \\
\hline Dipeptide & 99 \\
\hline Gamma-glutamyl amino acid & 4 \\
\hline Polypeptide & 3 \\
\hline Xenobiotics & 71 \\
\hline Benzoate & 4 \\
\hline Chemical & 13 \\
\hline Drug & 10 \\
\hline Food component/plant & 33 \\
\hline Xanthine & 11 \\
\hline
\end{tabular}

has been increased in high fat diet-induced obesity, which is an elevated CRC risk factor [32]. N125 was also shown as diet-modifiable in urinary metabolomes [28], supporting its possible importance as a dietmodifiable mediator of CRC pathogenesis. Additionally, excess sorbitol has been associated with diabeticrelated microvascular complications and retinopathy, and its accumulation was associated with osmotic and oxidative stress damage to the endothelium [30].

Beyond specific metabolite differences, we also evaluated entire metabolic networks to interrogate their contributions to both CRC and mucosal tissue microenvironments. The perturbation of short-chain fatty acid metabolism between CRC and adjacent mucosa is noteworthy because of the breadth of short-chain fatty acid metabolites emphasized in CRC prevention and control research. Short-chain fatty acids such as butyrate, propionate, and valerate have been shown to cause growth arrest and differentiation in human colorectal cancer cells [33]. In our study, short-chain 


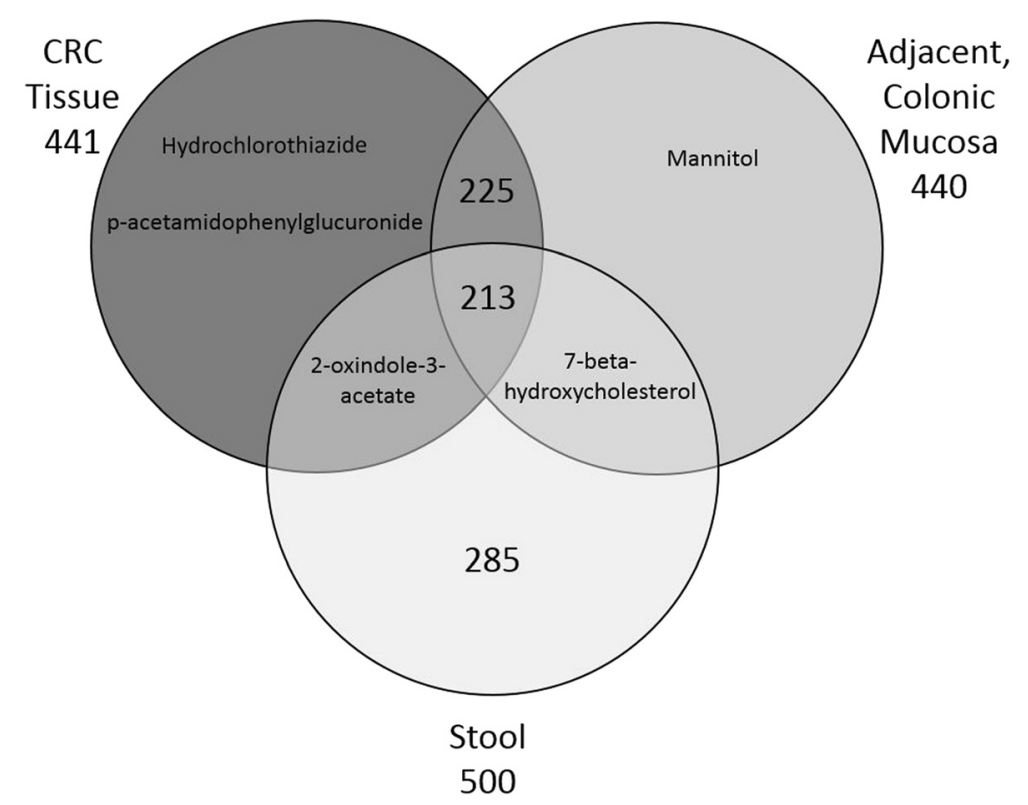

Fig. 4 Venn diagram of the total number of metabolites detected across CRC, adjacent mucosa, and stool samples from $(n=17)$ colorectal cancer patients. Nearly all metabolite detections were shared across CRC and adjacent mucosal tissue. Two-hundred fifteen metabolites from the stool metabolome were also detected in tissue matrices

fatty acid metabolism had a pathway impact score of 23.32 and contained the discriminatory metabolite valerate. These results taken together merit continued metabolomics investigation of short-chain fatty acids in the context of CRC pathogenesis and prevention, specifically valerate [34-36]. In addition to short-chain fatty acid metabolism, our pathway analysis revealed linkages to the glycolytic/gluconeogenic pathway and CRC tumorigenesis (pathway impact score of 5.8). This metabolic pathway contained 2 discriminatory metabolites: fructose-6phosphate and glucose-6-phosphate. Glucose-6phosphate and fructose-6-phosphate represent metabolites/intermediates of cellular respiration that are established for enhancing cancer cell energy required for rapid proliferation [37]. In this study, we observed depleted glucose-6-phosphate and fructose-6-phosphate levels in tumor tissues relative to adjacent mucosa which implies a Warburg effect or a switch from oxidative to substrate-level phosphorylation for energy production [38]. Larger cohort investigations may be needed to establish the signatures associated with short-chain fatty acid metabolism, fructose, mannose, and galactose metabolism, glycolysis, gluconeogenesis, and pyruvate metabolism, alanine and aspartate metabolism, leucine, isoleucine, and valine metabolism, and secondary bile acid metabolism (Fig. 2). Given that the CRC metabotype has not yet been expanded to provide metabolite information specific to the colonic location affected (i.e., proximal, distal, rectum), future investigations should consider this to be another experimental parameter by which to discriminate samples from each other and for enhanced precision in biomarker discovery [8].

Of the 703 metabolites detected in this study, $29.2 \%$ of metabolites overlap across tissue and stool matrices (Fig. 4). Colorectal tissue and stool metabolite profiles derive from several inputs including altered gene expression, oxidative stress responses, xenobiotic metabolism, and the utilization of alternative carbon sources from the gut microbiota. Findings from this pilot investigation of CRC and stool raises awareness regarding the utility of stool metabolites as relevant indicators of CRC tissue microenvironment $[39,40]$.

\section{Conclusions}

This pilot, global, non-targeted metabolome study identified various metabolic alterations in both individual metabolites and metabolic pathway networks between CRC and adjacent mucosa from CRC patients. In addition, we observed a low percentage of conserved detection across tissue and stool matrices (213 shared metabolites of 728 total metabolites; $29.2 \%$ conserved metabolites). Findings from this study reiterate the complexity of CRC biology with regard to individual phenotypes and the utility for a broad-spectrum metabolite detection platform to guide our approaches to treatment, control, and prevention of this complex malignancy. 


\section{Additional file}

Additional file 1: 2016 metabolomics investigation supplemental file. Supplemental Table S1. Supplemental Table S2. Supplemental Figure S1. (DOCX $1332 \mathrm{~kb})$

\section{Abbreviations}

ANOVA, analysis of variance; APC, adenomatous polyposis coli; BRAF, B-Raf proto-oncogene, serine/threonine kinase; CEA, carcinoembryonic antigen: COX, cyclooxygenase; CRC, colorectal cancer; FD, fold difference; GC/MS, gas chromatography mass spectrometry; HMDB, human metabolome database; KEGG, Kyoto Encyclopedia Genes and Genomes; KRAS, Kirsten rat sarcoma oncogene homolog; LC-MS/MS, liquid chromatography mass spectrometry; m/z, mass-to-charge ratio; MPA, MetaboLync pathway analysis; MSI, microsatellite instability; NSAID, non-steroidal anti-inflammatory drugs; $\mathrm{PVH}$, Poudre Valley Hospital; RI/RT, retention index/retention time.

\section{Acknowledgements}

The authors would like to thank the Poudre Valley Hospital/Oncology Clinical Research team, Erica C. Borresen, and Brittany Barnett for their technical and logistical support of this work. We would also like to thank Dr. Stephen Pettine, and the surgeons of the Northern Colorado Surgical Associates, who participated in recruitment and sample collection. The authors would like to acknowledge pilot project funding from the University of Colorado Cancer Center, Division of Cancer Control and Prevention, an NIH RO3CA150070-2 award, and interna department funds (Ryan) from Environmental and Radiological Health Sciences at Colorado State University for supporting this project.

\section{Availability of data and materials}

The data sets supporting the results of this article are included within the article and its additional files.

\section{Authors' contributions}

DB participated in result interpretations, metabolome data analysis, and wrote the manuscript. SR performed the statistical analyses for the study. TW participated in study design, collection of samples for the study, and provided written comments for manuscript. JO and MB participated in patient recruitment, consent, and coordination of sample collection. RB helped coordinate the patient recruitment and helped with the study design. ER conceived the study design and coordinated the regulatory approvals, result interpretations, and writing of the manuscript. All authors read and approved the final manuscript.

\section{Competing interests}

The authors declare that they have no competing interests.

\section{Author details}

'Department of Environmental and Radiological Health Sciences, Colorado State University, 200 West Lake Street, 1680 Campus Delivery, Fort Collins, CO 80523, USA. ${ }^{2}$ Department of Clinical Sciences, Colorado State University, Fort Collins, CO 80523, USA. ${ }^{3}$ Department of Food Science and Human Nutrition, Colorado State University, Fort Collins, CO 80523, USA. ${ }^{4}$ University of Colorado Health-North, Fort Collins, CO 80522, USA. ${ }^{5}$ Division of Medical Oncology, University of Colorado School of Medicine, Aurora, CO 80045, USA.

\section{Received: 26 January 2016 Accepted: 16 May 2016}

\section{Published online: 06 June 2016}

\section{References}

1. Lech G, Slotwinski R, Krasnodebski IW. The role of tumor markers and biomarkers in colorectal cancer. Neoplasma. 2013. doi:10.4149/neo_ 2014_003.

2. Siegel R, Desantis C, Jemal A. Colorectal cancer statistics. CA Cancer J Clin. 2014;64(2):104-17. doi:10.3322/caac.21220

3. Phua LC, Chue XP, Koh PK, Cheah PY, Ho HK, Chan EC. Non-invasive fecal metabonomic detection of colorectal cancer. Cancer Biol Ther. 2014;15(4):389-97. doi:10.4161/cbt.27625.

4. Ned RM, Melillo S, Marrone M. Fecal DNA testing for colorectal cancer screening: the ColoSure test. PLoS currents. 2011;3, RRN1220. doi:10.1371/currents.RRN1220.
5. Zou M, Zhang PJ, Wen XY, Chen L, Tian YP, Wang Y. A novel mixed integer programming for multi-biomarker panel identification by distinguishing malignant from benign colorectal tumors. Methods. 2015. doi:10.1016/j. ymeth.2015.05.011.

6. Schmidt CW. Metabolomics: what's happening downstream of DNA. Environ Health Perspect. 2004;112(7):A410-5.

7. Shirakami Y, Shimizu M, Kubota M, Araki H, Tanaka T, Moriwaki H, et al. Chemoprevention of colorectal cancer by targeting obesity-related metabolic abnormalities. World J Gastroenterol. 2014;20(27):8939-46. doi:10.3748/wjg.v20.i27.8939.

8. Chan EC, Koh PK, Mal M, Cheah PY, Eu KW, Backshall A, et al. Metabolic profiling of human colorectal cancer using high-resolution magic angle spinning nuclear magnetic resonance (HR-MAS NMR) spectroscopy and gas chromatography mass spectrometry (GC/MS). J Proteome Res. 2009;8(1): 352-61. doi:10.1021/pr8006232.

9. Qiu Y, Cai G, Zhou B, Li D, Zhao A, Xie G, et al. A distinct metabolic signature of human colorectal cancer with prognostic potential. Clini Cancer Res. 2014;20(8):2136-46. doi:10.1158/1078-0432.CCR-13-1939.

10. Yamane LS, Scapulatempo-Neto C, Alvarenga L, Oliveira CZ, Berardinelli GN, Almodova $\mathrm{E}$ et al. KRAS and BRAF mutations and MSI status in precursor lesions of colorectal cancer detected by colonoscopy. Oncology reports. 2014. doi:10.3892/or.2014.3338

11. Williams MD, Reeves R, Resar LS, Hill Jr HH. Metabolomics of colorectal cancer: past and current analytical platforms. Anal Bioanal Chem. 2013; 405(15):5013-30. doi:10.1007/s00216-013-6777-5.

12. Donohoe DR, Collins LB, Wali A, Bigler R, Sun W, Bultman SJ. The Warburg effect dictates the mechanism of butyrate-mediated histone acetylation and cell proliferation. Mol Cell. 2012;48(4):612-26. doi:10.1016/j.molcel.2012.08.033

13. Assfalg M, Bertini I, Colangiuli D, Luchinat C, Schafer H, Schutz B, et al. Evidence of different metabolic phenotypes in humans. Proc Natl Acad Sci U S A. 2008;105(5):1420-4. doi:10.1073/pnas.0705685105.

14. Montrose DC, Zhou XK, Kopelovich L, Yantiss RK, Karoly ED, Subbaramaiah K, et al. Metabolic profiling, a noninvasive approach for the detection of experimental colorectal neoplasia. Cancer Prev Res. 2012;5(12):1358-67. doi:10.1158/1940-6207.CAPR-12-0160

15. Park JS, Choi GS, Hasegawa S, Sakai Y, Huh JW, Kim HR, et al. Validation of the seventh edition of the American Joint Committee on cancer tumor node-staging system in patients with colorectal carcinoma in comparison with sixth classification. J Surg Oncol. 2012;106(6):674-9. doi:10.1002/jso.23117.

16. DeHaven CDE, Anne M, Dai H, Lawton KA. Software techniques for enabling high-throughput analysis of metabolomic datasets. InTech. 2012

17. Evans AM, DeHaven CD, Barrett T, Mitchell M, Milgram E. Integrated, nontargeted ultrahigh performance liquid chromatography/electrospray ionization tandem mass spectrometry platform for the identification and relative quantification of the small-molecule complement of biological systems. Anal Chem. 2009:81(16):6656-67. doi:10.1021/ac901536h.

18. Sumner LW, Amberg A, Barrett D, Beale MH, Beger R, Daykin CA, et al. Proposed minimum reporting standards for chemical analysis Chemical Analysis Working Group (CAWG) Metabolomics Standards Initiative (MSI). Metabolomics: Official journal of the Metabolomic Society. 2007;3(3):211-21. doi:10.1007/s11306-007-0082-2.

19. Team RC. R: A language and environment for statistical computing. Vienna Austria: R Foundation for Statistical Computing; 2013.

20. Smoot ME, Ono K, Ruscheinski J, Wang PL, Ideker T. Cytoscape 2.8: new features for data integration and network visualization. Bioinformatics. 2011; 27(3):431-2. doi:10.1093/bioinformatics/bta675.

21. Guo L, Milburn MV, Ryals JA, Lonergan SC, Mitchell MW, Wulff JE, et al. Plasma metabolomic profiles enhance precision medicine for volunteers of normal health. Proc Natl Acad Sci U S A. 2015:112(35):E4901-10. doi:10.1073/pnas.1508425112.

22. Goedert JJ, Sampson JN, Moore SC, Xiao Q, Xiong X, Hayes RB, et al. Fecal metabolomics: assay performance and association with colorectal cancer. Carcinogenesis. 2014;35(9):2089-96. doi:10.1093/carcin/bgu131.

23. Williams MD, Zhang X, Park JJ, Siems WF, Gang DR, Resar LM et al. Characterizing metabolic changes in human colorectal cancer. Analytical and bioanalytical chemistry. 2015. doi:10.1007/s00216-015-8662-X.

24. Qin P, Tang X, Elloso MM, Harnish DC. Bile acids induce adhesion molecule expression in endothelial cells through activation of reactive oxygen 
species, NF-kappaB, and p38. Am J Physiol Heart Circ Physiol. 2006;291 (2): H741-7. doi:10.1152/ajpheart.01182.2005.

25. Bernstein H, Bernstein C, Payne CM, Dvorak K. Bile acids as endogenous etiologic agents in gastrointestinal cancer. World J Gastroenterol. 2009; 15(27):3329-40.

26. Ajouz H, Mukherji D, Shamseddine A. Secondary bile acids: an underrecognized cause of colon cancer. World I Surg Oncol. 2014;12:164. doi:10.1186/1477-7819-12-164.

27. Song S, Guha S, Liu K, Buttar NS, Bresalier RS. COX-2 induction by unconjugated bile acids involves reactive oxygen species-mediated signalling pathways in Barrett's oesophagus and oesophageal adenocarcinoma. Gut. 2007;56(11):1512-21. doi:10.1136/gut.2007.121244.

28. Ryan EP, Heuberger AL, Broeckling CD, Borresen EC, Tillotson C, Prenni JE. Advances in nutritional metabolomics. Current Metabolomics. 2013;:109-20.

29. Christl SU, Bartram HP, Ruckert A, Scheppach W, Kasper H. Influence of starch fermentation on bile acid metabolism by colonic bacteria. Nutr Cancer. 1995;24(1):67-75. doi:10.1080/01635589509514394.

30. Kinoshita JH, Nishimura $\mathrm{C}$. The involvement of aldose reductase in diabetic complications. Diabetes Metab Rev. 1988;4(4):323-37.

31. Rutkowski B, Slominska E, Szolkiewicz M, Smolenski RT, Striley C, Rutkowski $P$, et al. N-methyl-2-pyridone-5-carboxamide: a novel uremic toxin? Kidney Int Suppl. 2003:84:S19-21. doi:10.1046/j.1523-1755.63.s84.36.x.

32. Boulange CL, Claus SP, Chou CJ, Collino S, Montoliu I, Kochhar S, et al. Early metabolic adaptation in C57BL/6 mice resistant to high fat diet induced weight gain involves an activation of mitochondrial oxidative pathways. J Proteome Res. 2013;12(4):1956-68. doi:10.1021/pr400051s.

33. Hinnebusch BF, Meng SF, Wu JT, Archer SY, Hodin RA. The effects of short-chain fatty acids on human colon cancer cell phenotype are associated with histone hyperacetylation. J Nutr. 2002;132(5):1012-7.

34. Louis P, Hold GL, Flint HJ. The gut microbiota, bacterial metabolites and colorectal cancer. Nat Rev Microbiol. 2014;12(10):661-72. doi:10.1038/nrmicro3344.

35. Weir TL, Manter DK, Sheflin AM, Barnett BA, Heuberger AL, Ryan EP. Stool microbiome and metabolome differences between colorectal cancer patients and healthy adults. PLoS One. 2013;8(8):e70803. doi:10.1371/journal.pone.0070803.

36. Hester CM, Jala VR, Langille MG, Umar S, Greiner KA, Haribabu B. Fecal microbes, short chain fatty acids, and colorectal cancer across racial/ ethnic groups. World J Gastroenterol. 2015;21(9):2759-69. doi:10.3748/wjg.v21.i9.2759.

37. Hamanaka RB, Chandel NS. Targeting glucose metabolism for cancer therapy. J Exp Med. 2012;209(2):211-5. doi:10.1084/jem.20120162.

38. Nam SO, Yotsumoto F, Miyata K, Fukagawa S, Yamada H, Kuroki M, et al. Warburg effect regulated by amphiregulin in the development of colorectal cancer. Cancer Med. 2015;4(4):575-87. doi:10.1002/cam4.416.

39. Wang H, Tso V, Wong C, Sadowski D, Fedorak RN. Development and validation of a highly sensitive urine-based test to identify patients with colonic adenomatous polyps. Clini Transl Gastroenterol. 2014;5:e54. doi:10.1038/ctg.2014.2.

40. Zamani Z, Arjmand M, Vahabi F, Eshaq Hosseini SM, Fazeli SM, Iravani A, et al. A metabolic study on colon cancer using (1) h nuclear magnetic resonance spectroscopy. Biochem Res Int. 2014;2014:348712. doi:10.1155/2014/348712

\section{Submit your next manuscript to BioMed Central and we will help you at every step:}

- We accept pre-submission inquiries

- Our selector tool helps you to find the most relevant journal

- We provide round the clock customer support

- Convenient online submission

- Thorough peer review

- Inclusion in PubMed and all major indexing services

- Maximum visibility for your research

Submit your manuscript at www.biomedcentral.com/submit
Biomed Central 